\title{
Genetic improvement of fungal pathogens
}

\section{What is a fungus?}

A eukaryotic, heterotrophic organism devoid of chlorophyll that obtains its nutrients by absorption, and reproduces by spores. The primary carbohydrate storage product of fungi is glycogen. Most fungi have a thallus composed of hyphae (sing. hypha) that elongate by tip growth.

\section{Associations}

Fungi are involved in symbiotic relationships with a number of organisms
i. Lichens
ii. Mycorrhizas
iii. Endophytes
iv. Mutualists

\section{Decomposers}

As saprotrophs, particularly as decomposers, fungi are essential components of the carbon cycle and are among the few organisms that can break down lignin.

\section{Groups of entomogenous fungi}
i. Phycomycetes
ii. Oomycetes

iii. Zygomycetes -Entomophthoraceae

iv. Deuteromycetes -Fungi imperfecti \}Most widely used for insect control

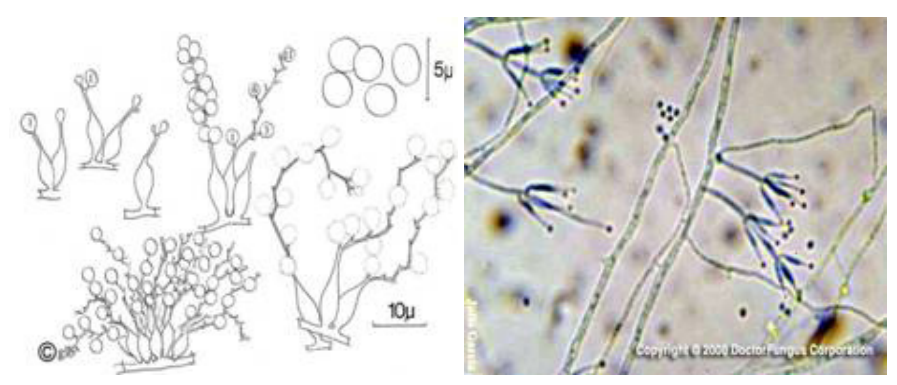

\section{Potential entomopathogenic fungi}

i. Metarhiziumspp.

ii. Beauveriaspp.

iii. Verticillium lecanii

iv. Nomuraea rileyi

v. Hirsutellaspp.

vi. Paecilomycesspp.

\section{Genetic improvement of EPF}

i. Chemical mutagenesis

\author{
Volume 8 Issue I - 2018 \\ P Parthiban,' RK Murali Baskaran,' \\ CChinniah,' ARavikumar,' K Thangavel' \\ 'Department of Agricultural Entomology, Tamil Nadu \\ Agricultural University, India \\ ${ }^{2}$ National Institute of Biotic Stress Management, India
}

Correspondence: P Parthiban, Department of Agricultural Entomology, Agricultural College and Research Institute,Tamil Nadu Agricultural University, Madurai-625 104,Tamil Nadu, India, Email parthitnau@gmail.com

Received: October 21, 2016 | Published: January 05, 2018 iii. Protoplast fusion

iv. Direct genetic manipulation

\section{Mutagen}

In genetics, a mutagen is a physical or chemical agent that changes the genetic material, usually DNA, of an organism and thus increases the frequency of mutations above the natural background level. As many mutations cause cancer, mutagens are therefore also likely to be carcinogens. Not all mutations are caused by mutagens: so-called "spontaneous mutations" occur due to spontaneous hydrolysis, errors in DNA replication, repair and recombination.

\section{Parasexual cycle}

The Parasexual cycle (or para sexuality), a process peculiar to fungi and single-celled organisms, is a nonsexual mechanism for transferring genetic material without meiosis or the development of sexual structures. ${ }^{1}$ It was first described by Italian geneticist Guido Pontecorvo in 1956 during studies on Aspergillus nidulans (also called Emericella nidulans when referring to its sexual form, or teleomorph). A Parasexual cycle is initiated by the fusion of hyphae (anastomosis) during which nuclei and other cytoplasmic components occupy the same cell (heterokaryosis and plasmogamy). Fusion of the unlike nuclei in the cell of the heterokaryon results in formation of a diploid nucleus (karyogamy), which is believed to be unstable and can produce segregants by recombination involving mitotic crossing over and tabloidization. Mitotic crossing-over can lead to the exchange of genes on chromosomes; while tabloidization probably involves mitotic non disjunctions which randomly reassert the chromosomes and result in the production of aneuploid and haploid cells.

Like a sexual cycle, para sexuality gives the species the opportunity to recombine the genome and produce new genotypes in their offspring. Unlike a sexual cycle, the process lacks coordination and is exclusively mitotic. The Parasexual cycle resembles sexual reproduction. In both cases, unlike hyphae (or modifications thereof) may fuse (plasmogamy) and their nuclei will occupy the same cell. The unlike nuclei fuse (karyogamy) to form a diploid (zygote) nucleus. In contrast to the sexual cycle, in the para sexual cycle recombination 
takes place during mitosis followed by tabloidization (but without meiosis). The recombined haploid nuclei appear among vegetative cells, which differ genetically from those of the parent mycelium. Both heterokaryosis and the para sexual cycle are very important for those fungi that have no sexual reproduction. Those cycles provide for somatic variation in the vegetative phase of their life cycles. This is also true for fungi where the sexual phase is present, although in this case, additional and significant variation is incorporated through the sexual reproduction.

\section{Protoplast fusion}

Somatic fusion, also called protoplast fusion, is a type of genetic modification in fungi.

\section{Genetic engineering}

Genetic engineering, also called genetic modification, is the direct manipulation of an organism's genome using biotechnology. New DNA may be inserted in the host genome by first isolating and copying the genetic material of interest using molecular cloning methods to generate a DNA sequence, or by synthesizing the DNA, and then inserting this construct into the host organism. Genes may be removed, or "knocked out", using a nuclease. Gene targeting is a different technique that uses homologous recombination to change an endogenous gene, and can be used to delete a gene, remove exons, add a gene, or introduce point mutations.

\section{EPF-strain improvement}

i. Parasexual recombination -B. bassiana x B. sulfurescens recombinants

ii. Direct gene manipulation -Ben A3 gene for Ben late resistance

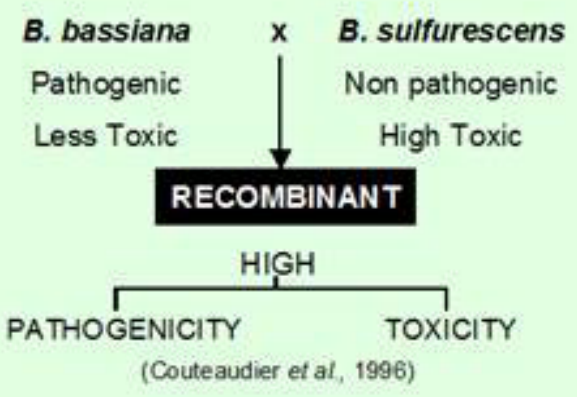

\section{Protoplast fusion technology}

Protoplasts are the cells of which cell walls are removed and cytoplasmic membrane is the outermost layer in such cells. Protoplast can be obtained by specific lytic enzymes to remove cell wall. Protoplast fusion is a physical phenomenon, during fusion two or more protoplasts come in contact and adhere with one another either spontaneously or in presence of fusion inducing agents. By protoplast fusion it is possible to transfer some useful genes such as disease resistance, nitrogen fixation, rapid growth rate, more product formation rate, protein quality, frost hardiness, drought resistance, herbicide resistance, heat and cold resistance from one species to another. Protoplast fusion an important tools in strain improvement for bringing genetic recombination and developing hybrid strains in filamentous fungi. Protoplast fusion has been used to combine genes from different organisms to create strains with desired properties. These are the powerful techniques for engineering of microbial strains for desirable industrial properties. Protoplast fusion would continue to be an existing area of research in modern biotechnology. This technique in the future will be one of the most frequently used research tools for tissue culturists, molecular biologists, biochemical engineers and biotechnologists.

Introduction: The protoplast includes the plasma lemma and everything contained within i.e. the entire cell without its inherent cellulosic cell wall. In protoplast technology, two genetically different protoplast isolated from the somatic cells and are experimentally fused to obtain para sexual hybrid protoplasts. The hybrid protoplast contained heteroplasoic cytoplasm and two fused parent nuclei. Fusion of protoplast is relatively a new versatile technique to induce or promote genetic recombination in a variety of prokaryotic and eukaryotic cells (Bhojwani et al. 1977). Protoplast fusion may be used to produce inter specific or even intergeneric hybrids. Protoplast fusion becomes an important tool of gene manipulation because it breakdown the barriers to genetic exchange imposed by conventional mating systems. Protoplast fusion technique has a great potential for genetic analysis and for strain improvement. It is particularly useful for industrially useful microorganisms (Murlidhar \& Panda 2000).

\section{Enzymes used for breaking of cell walls}

For protoplast fusion it is important that the cell wall of plant and microorganisms is degraded .So various enzymes used for this process. Cellulase and pectinase or macerozyme acting on plant cell wall. Bacterial cell walls are degraded by the action of lysozyme. Fungal wall degraded by Novozyme-234 which includes glucanase and chitinase (Narayanswamy 1994; Jogdand 2001).

Methods of protoplast fusion: Protoplast fusion can be broadly classified into two categories.

Spontaneous fusion: Protoplast during isolation often fuses spontaneously and this phenomenon is called spontaneous fusion. During the enzyme treatment, protoplast from adjoining cells fuses through their plasmodesmata to form multinucleate protoplasts.

Induced fusion: Fusion of freely isolated protoplasts from different sources with the help of fusion inducing chemicals agent $\mathrm{s}$ is known as induced fusion. Normally isolated protoplast do not fuse with each other because the surface of isolated protoplast carries negative charges $(-10 \mathrm{mV}$ to- $30 \mathrm{mV})$ around the outside of the plasma membrane. And thus there is a strong tendency in the protoplast to repel each other due to their same charges .So this type of fusion needs a fusion inducing chemicals which actually reduce the electro negativity of the isolated protoplast and allow them to fuse with each other's (Narayanswamy 1994). The isolated protoplast can be induced to fuse by three ways.

Mechanical fusion: In this process the isolated protoplast are brought into intimate physical contact mechanically under microscope using micromanipulator or perfusion micropipette.

Chemo fusion: Several chemicals have been used to induce protoplast fusion such as sodium nitrate, polyethylene glycol, Calcium ions $(\mathrm{Ca}++)$. Chemical fusogens cause the isolated protoplast to adhere each other and leads to tight agglutination followed by fusion of protoplast. ${ }^{2}$ In order to convert cellulosic materials to ethanol by single step process, ${ }^{3}$ carried out chemo fusion between protoplasts of Trichoderma reesei QM9414 and Saccharomyces cerrevesei NCIM 
3288. Observed successful fusion suggest that endo glucanase is the key enzyme in the success of fusion (Iwata et al. 1986), were obtained Tetracycline resistant (Tetr), erythromycin resistant (Eryr) fusants of Lactobacillus fermentatum 604 carrying a 10 megadalton Tetr plasmid and L. fermentatum 605 carrying a 38 megadalton Eryr plasmid by means of polyethylene glycol induced protoplast fusion. Chemo fusion is a non specific, inexpensive, can cause massive fusion product, can be cytotoxic and non selective and having less fusion frequency.

Electro fusion: Recently, mild electric stimulation is being used to fuse protoplast. In this two glass capillary microelectrode are placed in contact with the protoplast. An electric field of low strength $(10 \mathrm{Kvm}-1)$ gives rise to di electrophoretic dipole generation within the protoplast suspension. This leads to pearl chain arrangement of protoplasts. Subsequent application of high strength of electric fields $(100 \mathrm{kvm}-1)$ for some microseconds results in electric breakdown of membrane and subsequent fusion (Ushishima et al. 1991; Jogdand 2001, Groth et al. 1987) carried out electofusion of Penicillium protoplasts, after di a electrophoresis and found viable fusion products. ${ }^{4}$ Reported electrically induced protoplast fusion using pulse electric field for di electrophoresis and suggest the possibility of electrically induced protoplast fusion at cation concentration that prevents fusion when sine-wave fields are applied. Gaint protoplast of Pleurotus cornucopiae was fused using the glass microelectrode fusion technique. To induce fusion $\mathrm{Ca}++$ was necessary .Polyethylene glycol 400 (PEG) promoted fusion but also increased the adhesion of protoplasts (Magae et al. 1986). In order to regulate electrofusion (Uran et al. 1998) studied electro fusion procedures for yeast breeding and reported that cell membrane fusion behaviour of respiration deficient yeasts (P-) was remarkably different from the normal yeasts $(\mathrm{P}+)$. Induction of cell membrane fusion in $\mathrm{P}+$ protoplast appeared under the pulse conditions (height $2.5-5.5 \mathrm{kVcm}-1$ and duration 25 $430 \mu \mathrm{s})$ and the time interval of morphological change from the long to short state was 3-11s. On the other hand, induction of cell membrane fusion in P- protoplasts appeared under the higher pulse condition (height: $4.0-7.0 \mathrm{kV} \mathrm{cm}-1$ and duration: $20-500 \mu \mathrm{s}$ ). The time interval of the morphological change from the long to short state was 110-170 $\mathrm{s}$ in cell membrane fusants of $\mathrm{P}$ - protoplasts. The i-potential of $\mathrm{P}+$ protoplasts was -10 to- $30 \mathrm{mV}$ and that of the P-protoplasts was-25-60 $\mathrm{mV}$. The surface charge of the P-protoplasts was more negative than that of $\mathrm{P}+$ protoplasts; therefore, regulation of electro fusion among various kinds of yeast strains was possible by changing the surface charge of the protoplasts using mitochondrial mutations. Electro fusion is easy to control having fusion frequency upto $100 \%$. Gives reproducibility; less cytotoxic. But equipment is sophisticated and expensive.

Mechanism of protoplast fusion: The mechanism of protoplast fusion is not fully known .Several explanations have been put forward to understand the mechanism of protoplast fusion. Some are explained here: When the protoplasts are brought into close proximity, this is followed an induction phase thereby changes induced in electrostatic potential of the membrane results in fusion. After the fusion, the membranes stabilize and the surface potential returns to their former state. Other literature showed when the protoplasts are closely adhered, the external fusogens cause disturbance in the intra membranous proteins and glycoproteins. This increases membrane fluidity and creates a region where lipid molecule intermix, allowing coalescence of adjacent membranes. The negative charge carried by protoplast is mainly due to intra membranous phosphate groups'.
The addition of $\mathrm{Ca}++$ ions causes reduction in the zeta potential of plasma membrane and under this situation protoplasts are fused. ${ }^{5}$ The high molecular weight polymer (1000-6000) of PEG acts as a molecular bridges connecting the protoplasts. Calcium ions linked the negatively charged PEG and membrane surface. On elution of the PEG, the surface potential are disturbed, leading to intra membrane contact and subsequent fusion, Besides this ,the strong affinity of PEG for water may cause local dehydration of the membrane and increase fluidity, thus inducing fusion. Protoplast fusion takes place when the molecular distance between the protoplasts is $10 \mathrm{~A}$ or less. This indicates that protoplast fusion is highly a traumatic event (Jogdand 2001; Narayanswamy 1994).

Protoplast fusion in fungi: Production and regeneration of protoplasts is a useful technique for fungal transformations. Commercial preparation of enzymes which contain mixture of products to digest fungal cell wall used. Novozyme 234 includes (glucanase and chitinase) enzyme mixture is added to rapidly growing fungal tissue suspensed in an osmotic buffer (e.g. 0.6 mol-1, KCl, $1.2 \mathrm{~mol}-1$, Sorbitol or $\left.1.2 \mathrm{~mol}-1 \mathrm{MgSO}_{4}\right)$. The protoplasts and DNA are mixed in presence of $15 \%(\mathrm{w} / \mathrm{V})$ PEG 6000 and $\mathrm{pH}$ buffer (TRIS HCl). 10mml-1. PEG causes clump formation in protoplasts. At $37^{\circ} \mathrm{C}$, grow mycelium on cello phone placed on agar overnight. Incubate with enzyme at $30^{\circ} \mathrm{C}$ for 1.5 hours in empty petridishes having $\mathrm{KCl}$, than filter protoplasts, wash protoplast in $\mathrm{KCl}$ (Centrifuge and re suspended the pellets). Protoplast fusion frequency in fungi is $0.2-2 \%,{ }^{3}$ (Jogdand 2001).

\section{Strain improvement of entomopathogenic fungal species beauveria bassiana and metarhizium anisopliae by protoplast fusion}

\section{EPF-strain improvement}

Entomopathogenic fungi exist as ubiquitous saprophyte in soil and often cause widespread epizootics, wiping out insect pest populations on agricultural crops. Conidia are the infective propagules and it is generally assumed that high humidity and optimum temperature are required for the fungus to germinate. Conidia upon contact adhere to insect cuticle under favourable conditions and germinate. The germ tubes secrete extracellular enzymes which breach the insect cuticle made of 75-80 \% protein. ${ }^{6}$ Proteinaceous outer integument of insect forms an effective barrier and proteases are the virulent factors that play a key role in degradation. ${ }^{7}$ Proteases from B. bassiana and M. anisopliae are capable of degrading wide variety of cuticle proteins. Serine proteases $\operatorname{Pr} 1$ and $\operatorname{Pr} 2$ have been identified in B. bassiana and M. anisopliae. ${ }^{8} \operatorname{Pr} 1$ belong to subtilisin like serine protease and Pr2 is a trypsin like serine protease. ${ }^{9}$ Like many fungi B. bassiana and M. anisopliae lack conventional sexual cycle as they are asexual forms and rely entirely on para sexual recombination. ${ }^{10}$ As in meiosis, segregation and crossing over of chromosomes occur in para sexual recombination but independent of one another. Fungal protoplast fusion has been established as a means to transfer genetic material and provides an effective method for genetic manipulation and strain improvement. ${ }^{11}$ High virulent hybrid strains of entomopathogenic fungi were obtained by certain researchers (Couteaudier et al. 1997). Successful fusion of protoplasts in Trichoderma and Aspergillus has been well demonstrated. ${ }^{12}$ Present study aims at strain improvement in entomopathogenic fungi which involves isolation and fusion of protoplasts from two different genera and performing interstrain, intra-strain and intergeneric fusions through para sexual recombination 


\section{Fungal cultures}

Beauveria bassiana strains UB1 (ARSEF 1788) isolated from Helicoverpa virescens in Spain is low extracellular protease producer and UB9 (ARSEF 2033) isolated from Cocinella spp. in U.S.A is high extracellular protease producer, Metarhizium anisopliae strains UM6 (ARSEF 2596) isolated from Pyrausta machaeralis in India is high extracellular protease producer and UM10 (ARSEF 3295) isolated from Anticarsia gemmatalis in Mexico is a low extracellular protease producing these strains were grown in Sabouraud's Dextrose [SD] broth (Dextrose-4 gm, Yeast extract-1 gm, Peptone-1 gm, distilled water- $100 \mathrm{ml}$ ) and incubated in a shaker for 40 hour at $28^{\circ} \mathrm{C}$.

\section{Isolation of protoplasts}

After 48 hour of incubation mycelium was harvested and centrifuged for $10 \mathrm{~min}$ at $8000 \mathrm{~g}$ and $4^{\circ} \mathrm{C}$. The mycelial pellet was washed twice with wash buffer and $1 \mathrm{ml}$ of lytic enzyme for $50 \mathrm{mg}$ of mycelium was added, incubated in a shaker at $28^{\circ} \mathrm{C}$ and $180 \mathrm{rpm}$ for 3 hour. The protoplasts were separated from mycelial debris by filtration through sterile $30 \mu \mathrm{m}$ nylon muslin and finally protoplasts were collected by centrifugation at $4000 \mathrm{~g}$ for $10 \mathrm{~min}$ and the pellet was dissolved in $200 \mu 1$ of $0.7 \mathrm{M} \mathrm{KCl}$.

\section{Fusion of protoplasts}

Intra-strain, inter-strain and inter-generic protoplast fusions were carried out by mixing $1 \times 107 / \mathrm{ml}$ protoplasts of each strain from B. bassiana (UB1\&UB9) and M. anisopliae (UM6\&UM10) by centrifugation. The pellet was incubated in $1 \mathrm{ml}$ of $20 \%$ PEG 6000 containing $0.01 \mathrm{M} \mathrm{CaCl}_{2}$ and $0.05 \mathrm{M}$ Glycine $(\mathrm{pH} 7.5)$ for $30 \mathrm{~min}$ at $28^{\circ} \mathrm{C}$. Parental protoplasts were subjected to fusion separately to same PEG treatment.

\section{Regeneration of fusants}

The PEG in the fusion mixture was washed away twice using wash buffer. Fused protoplasts each strain by centrifugation at $5000 \mathrm{~g}$ for $10 \mathrm{~min}$ and were suspended in $0.6 \mathrm{M}$ potassium phosphate buffer. Protoplasts were placed on Czapekdox agar (5gm Glucose, $0.1 \mathrm{gm} \mathrm{KH}_{2} \mathrm{PO}_{4}, 0.2 \mathrm{gm} \mathrm{NaNO}, 0.025 \mathrm{gm} \mathrm{MgSO}$. $7 \mathrm{H}_{2} \mathrm{O}, 0.001 \mathrm{gm}$ $\mathrm{FeSO}_{4} \cdot 7 \mathrm{H}_{2} \mathrm{O}, 5.22 \mathrm{gm} \mathrm{KCl}, 2 \mathrm{gm} \mathrm{Agar}, 100 \mathrm{ml} \mathrm{DW}$ ) for regeneration process. Agar plates were incubated for 3-4 days at $28^{\circ} \mathrm{C} .100 \mu \mathrm{l}$ of the suspension was taken for microscopic observation and photographs were taken using trinocular microscope. After 3-4days of incubation appearance of colonies was observed and regeneration frequency was determined by using the formula:

Regeneration frequency $=$ Number of colonies regenerated $\mathrm{x} 100$ Number of protoplasts inoculated

\section{Sub culturing of regenerated protoplasts}

Fast growing colonies from agar plates were selected randomly and sub cultured by streaking on Sabouraud's Dextrose [SD] agar slants (4gm Dextrose, $1 \mathrm{gm}$ Yeast extract 1gm Peptone, 2gm Agar, 100ml DW) and incubated at $28^{\circ} \mathrm{C}$ for 2-3days under sterile conditions. Fully grown colonies were used for the production of protease and tested for its enzymatic activity.

\section{Cultivation of entomopathogenic fungi for protease production}

The fungal colonies from regenerated slants were inoculated into
MM amended with casein and incubated for 72 hour at $28^{\circ} \mathrm{C}$. The culture was centrifuged for $10 \mathrm{~min}$ at $4^{\circ} \mathrm{C}$ and $8000 \mathrm{~g}$. Supernatant was taken as crude enzyme and stored at $4^{\circ} \mathrm{C}$ for further assay.

\section{Proteolytic assay}

Casein substrate $(400 \mu l)$ was added to $200 \mu$ l of crude enzyme. Later $200 \mu 1$ of $0.01 \mathrm{M}$ Tris $\mathrm{HCl}(\mathrm{pH} 8.0)$ was added to the mixture, incubated at $37^{\circ} \mathrm{C}$ for $10 \mathrm{~min}$ and $100 \mu \mathrm{l}$ of $1.2 \mathrm{M}$ TCA was added to stop the reaction. Blank was prepared by adding TCA just before the addition of enzyme and absorbance of all the solutions were measured at $280 \mathrm{~nm}$.

\section{Assay of PrI \& Pr2}

Two synthetic substrates mainly N-Succinyl -Alanine prophepanilide and $\mathrm{N}-\alpha$ - Benzoyl- DL- Arginine $\mathrm{p}$-nitroanilide were used for the assay of $\operatorname{Pr} 1 \& \operatorname{Pr} 2$. The above mentioned synthetic substrates $(0.05 \mathrm{ml})$ were added to $0.85 \mathrm{ml}$ of $15 \mathrm{mM}$ Tris $\mathrm{HCl}(\mathrm{pH}$ 8.5 ) and $0.1 \mathrm{ml}$ of crude enzyme from each strain, mixed well and incubated for 1 hour at $28^{\circ} \mathrm{C}$.

\section{Genetic Nature, Stability, and Improved Virulence of Hybrids from Protoplast Fusion in Beauveria}

Genetic improvement of two different strains of the entomopathogenic fungus Beauveria bassiana for more effective control of Ostrinia nubilalis and Leptinotarsa decemlineata was obtained by crosses with the insecticidal toxin-producing strain Beauveria sulfurescens. Protoplast fusion between diauxotrophic mutants resulted in the recovery of some stable proto trophic fusion products. The low levels of virulence of the wild type strain B. bassiana 28 isolated originally from L. decemlineata were enhanced both on L. decemlineata and O. nubilalis for one of the hybrids obtained (FP8) from the cross B. bassiana $28 \times$ B. sulfurescens 2. Fusion product 25 obtained from the cross between B. sulfurescens and the highly pathogenic strain B. bassiana 147 showed a three-day reduction in the LT50 towards O. nubilalis. Southern blot hybridization with nine probe-enzyme combinations was conducted on genomic DNAs from the original wild strains, parental mutant strains, and fusion products. Additive banding patterns or unique banding pattern of either parental strain was observed in five hybrids, indicating their status as recombinant and/or partially diploid. Combination of RFLP markers indicative of both parental genomes was never observed with fusion product FP $25 . .^{13-15}$ The stability of the virulence following passage through insect-host and stability of molecular structure for the fusion products FP 8 and FP 25 suggest that asexual genetic recombination by protoplast fusion may provide an attractive method for the genetic improvement of biocontrol efficiency in entomopathogenic fungi.

\section{A new method for producing hybrid strains of the entomopathogenic fungus verticillium lecanii (Lecanicillium Spp.) through protoplast fusion by using nitrate non- utilizing (NIT) mutants}

Nitrate non-utilizing (nit) mutants are commonly used for determining vegetative compatibility groups (VCGs) through complementation testing in several fungal species. Major advantages of using nit mutants are that they can be easily obtained by selection on media containing chlorate, and that it is possible to recover mutants 
without mutagenic treatment as a result of spontaneous mutation. V. lecanii belongs to the Hyphomycetes, and it is not known to possess sexual stages. Hyphal anastomosis and heterokaryosis are the only mechanisms of nuclear migration among different strains of the same species. A parasexual cycle provides possibilities for breeding important industrial Hyphomycetes; however, vegetative compatibility impedes this process. This impediment can be overcome by protoplast fusion.

Protoplast fusion experiments by using Vertalec, Mycotal, and B-2 with nit mutants as genetic markers in order to obtain hybrid strains of V. lecanii. This is the first study that has utilized nit mutants for the visualization and genetic marking of fungal protoplast fusion. Subsequently, genomic DNA and mitochondrial DNA (mtDNA) of the hybrid strains were analyzed to assess protoplast fusion with respect to the genotype, and to compare it with that of the parental strains. Fungal strains and the generation of nit mutants. This study used three strains of V. lecanii. Vertalec and Mycotal were used as commercial biological control agents (BCAs) against the greenhouse aphid and whitefly. These strains had originated from single spores that were isolated from commercial preparations obtained from Koppert Biological Systems (Netherlands). The other strain was B-2 (MAFF238429, Ministry of Agriculture, Forestry and Fisheries of Japan), which has high colonization ability on cucumber leaves under conditions of low relative humidity. The nit mutants were generated using water agar chlorate (WAC) medium containing (per liter of distilled water) $2 \%$ agar, $0.02 \%$ glucose, and $2 \% \mathrm{KClO}_{3}$.

Mycelial plugs of Vertalec, Mycotal and B-2 that were quarried from the fringes of the respective colonies were transferred to the WAC medium, and incubated in darkness at 24C. Fast-growing sectors of chlorate-resistant colonies were transferred to a minimal medium (MM; basal medium (BM)+2g L-1 NaNO3). BM consisted of (per liter of distilled water) $30 \mathrm{~g}$ sucrose, $1 \mathrm{~g} \mathrm{KH}_{2} \mathrm{PO}_{4}, 0.5 \mathrm{~g} \mathrm{\textrm {MSO } _ { 4 }}$. $\mathrm{H}_{2} \mathrm{O}$, $0.5 \mathrm{gKCl}, 10 \mathrm{mgFeSO}_{4} .7 \mathrm{H}_{2} \mathrm{O}$, and $20 \mathrm{~g}$ agar; and $0.2 \mathrm{ml}$ trace element solution (per $100 \mathrm{ml}$ of distilled water) composed of $5 \mathrm{~g}$ citric acid, $5 \mathrm{~g}$ $\mathrm{ZnSO}_{4} \cdot 7 \mathrm{H}_{2} \mathrm{O}, 1 \mathrm{~g} \mathrm{Fe}\left(\mathrm{NH}_{4}\right) 2\left(\mathrm{SO}_{4}\right) 2.6 \mathrm{H}_{2} \mathrm{O}, 0.25 \mathrm{~g} \mathrm{CuSO}_{4} .5 \mathrm{H}_{2} \mathrm{O}, 50 \mathrm{mg}$ $\mathrm{MnSO}_{4}$. $\mathrm{H}_{2} \mathrm{O}, 50 \mathrm{mg} \mathrm{H}_{3} \mathrm{BO}_{4}$, and $50 \mathrm{mg} \mathrm{Na} \mathrm{MoO}_{4} \cdot 2 \mathrm{H}_{2} \mathrm{O}$. the plates (90 mm diameter) were then incubated for 10days. Thin expansive colonies with non-aerial mycelia were identified as nit mutants. Physiological phenotypes of nit mutants were detected by incubation in five media containing different nitrogen sources: nitrate medium $(\mathrm{MM})$, nitrite medium $\left(\mathrm{BM}+0.5 \mathrm{~g} \mathrm{~L}-1 \mathrm{NaNO}_{2}\right)$, hypoxanthine medium $(\mathrm{BM}+0.2 \mathrm{~g} \mathrm{~L}-1$ hypoxanthine), ammonium medium $(\mathrm{BM}+0.2 \mathrm{~g} \mathrm{~L}-1$ ammonium tartrate), and uric acid medium (BM+0.2g L-1 uric acid). Mycelial plugs of the nit mutants were placed in each of the five media, and incubated for 6-10days. Subsequently, colony morphology of phenotypes was evaluated by using the method of Correll et al. 1998. These evaluations were performed twice. Complementation was evaluated by a dual culture technique on MM plates, and it was ascertained if an aerial mycelium was formed at the contact zone between two distinct nit mutant. ${ }^{16-18}$

\section{Protoplast formation and fusion experiments}

For protoplast formation, conidia of the parental nit mutants were treated with $5 \mathrm{ml}$ of an enzyme solution [Novozyme 188 (Cellobiose from Aspergillus niger: Sigma Aldrich), 0.01g ml-1; and Lysing Enzymes from Trichoderma harzianum (containing $\beta$-glucanase, cellulase, protease, and chitinase activities: Sigma Aldrich), 0.01g $\mathrm{ml}-1$; in $1 \mathrm{M} \mathrm{MgSO}_{4}$ ] for $3-5 \mathrm{~h}$ at $30^{\circ} \mathrm{C}$ in a water bath incubator (50cycles/min). Subsequently, the protoplasts were centrifuged at $2000 \mathrm{rpm}$ for $5 \mathrm{~min}$. Pellets were resuspended in sorbitol solution [1 M sorbitol, $50 \mathrm{mM} \mathrm{CaCl}_{2}, 10 \mathrm{mM}$ Tris- $\left.\mathrm{HCl}(\mathrm{pH} 7.4)\right]$, and were further adjusted to ca. $1 \times 106$ protoplasts $\mathrm{ml}-1$.

Each protoplast suspension of nit1 and NitM was mixed, treated with prewarmed $\left(30^{\circ} \mathrm{C}\right) 30 \%$ polyethylene glycol (PEG) 4000 $[10 \mathrm{mM} \mathrm{CaCl}, 10 \mathrm{mM}$ Tris- $\mathrm{HCl}(\mathrm{pH} 7.5)]$, and incubated at $30^{\circ} \mathrm{C}$ for $15 \mathrm{~min}$. This mixture was centrifuged at $1000 \mathrm{rpm}$ for $5 \mathrm{~min}$. It was then resuspended in the sorbitol solution and plated on a MM plate. The detected phototrophic colonies were considered to be derived by protoplast fusion. Each colony was re-transferred on to the MM plate for purification. The only mycelia that demonstrated prototrophic growth on the MM plate were the single spore isolated colonies. Only stable isolates that were obtained after more than 20 generations of growth were transferred on to potato sucrose agar (200g boiled potato extract, $20 \mathrm{~g}$ sucrose, and $30 \mathrm{~g}$ agar per $1 \mathrm{~L}$ of distilled water) supplemented with $1 \%$ chitin (PSCA) as the hybrid strains. Mycelial plugs of hybrid strains and parental nit mutants were inoculated in the center of the plates $(90 \mathrm{~mm}$ diameter) containing PSCA, and were subsequently incubated as described above for approximately 2 weeks. Colony morphology was visually evaluated considering the color, shape, and quantity of aerial mycelia.

\section{Acknowledgements}

None.

\section{Conflict of Interest}

The author declares no conflict of interest.

\section{References}

1. Bondkly El AM. Gene Transfer between Different Trichoderma Species and Aspergillus niger through intergeneric protoplast fusion to convert ground rice straw to citric acid and cellulase. Appl Biochem Biotechnol. 2006;135(2):117-132.

2. Pasha C, Kuhad RC, Rao LV. Strain improvement of thermotolerant Saccharomyces cerevisiae VS strain for better utilization of lignocellulosic substrates. J Appl Microbiol. 2007;103(5):1480-1489.

3. Dimitrova AP, Christov AM. electrically induced protoplast fusion using pulse electric fields for die electrophoresis. Plant Physiol. 1992;100:2008-2012.

4. Anne J, Peberdy JF. Conditions for induced fusion of fungal protoplasts in polyethylene glycol solutions. Arch Microbiol. 1975;105(3):201-205.

5. Srinivas R, Panda T. Localization of carboxy methyl cellulase in the intergeneric fusants of Trichodermma reesei QM 9414 and Saccharomyces cerevisee NCIM 3288. Bioprocess Biosystems Engg. 1997;18:71-73.

6. Pekrul, Grula EA. Mode of infection of the corn earworm (Heliothis zea) by Beauveria bassianaas revealed by scanning electron microscopy. Journal of Invertebrate Pathology. 1979;34(3):238-247.

7. Clarkson, Charnley AK. New insights into the mechanisms of fungal pathogenesis in insects. Trends Microbiol. 1996;4(5):197-203.

8. Leger, Charnley AK, Cooper M. Distribution of chymoelastases and trypsin-like enzymes in five species of entomopathogenic deuteromycetes. Arch Biochem Biophys. 1987;258(1):123-131.

9. Leger. The role of cuticle-degrading proteases in fungal pathogenesis of insects. Canadian Journal of Botany. 1995;73(S1):1119-1125. 
10. Paccola Meirelles, Azevedo JL. Parasexuality in Beauveria bassiana Journal of Invertebrate Pathology. 1991;57(2):172-176.

11. Stasz, Harman GE, Gullino ML. Limited vegetative compatibility following intra and inter-strain protoplast fusion in Trichodermaspp. Experimental Mycology. 1989;13(4):364-371.

12. Avram D, Petcu I, Radu M, et al. Electrically induced protoplast fusion for ergosterol producing yeast strain improvement. J Basic Microbiol. 1992;32(6):369-372.

13. Brume MD, Parker VG, Alacemi M, et al. Strain improvement of Claviceps purpurea by protoplast fusion without introducing auxotrophic markers. Appl Microbiol Biotechnol. 1992;38(6):746-749.

14. Gokhale DV, Puntambekar US, Deobagkar DN. Protoplast fusion: A tool for intergeneric transfer in bacteria. Biotechnol Adv. 1993;11:199-217.
15. Mrinalini, Lalitha Kumari D. Integration of Enhanced biocontrol efficacy and fungicide tolerance in Trichoderma spp., by electro fusion. Journal of Plant Diseases. 1998;105(1):34-40.

16. Rubinder K, Chadha BS, Singh S, et al. Amylase hyper producing haploid recombinant strains of Thermomyces lanuginosusobtained by intraspecific protoplast fusion. Can J Microbiol. 2000;46(7):669-673.

17. Tschen, Li IF. Optimization of formation and regeneration of protoplast from biocontrol agents of Trichoderma spp. Mycoscience. 1997;35(3):257-263.

18. Zonneveld. Inhibiting effect of 2-deoxyglucose on cell wall $\alpha 1,3-$ glucan synthesis and Cleitothecium development of Aspergillus nichulans. Developmental Biology. 1973;34(1):1-8. 